\title{
Osteopontin deficiency protects against obesity-induced hepatic steatosis and attenuates glucose production in mice
}

\author{
F. W. Kiefer • S. Neschen • B. Pfau • B. Legerer • A. Neuhofer • M. Kahle • \\ M. Hrabé de Angelis • M. Schlederer • M. Mair • L. Kenner • J. Plutzky • M. Zeyda • \\ T. M. Stulnig
}

Received: 19 December 2010 / Accepted: 4 April 2011 /Published online: 12 May 2011

(C) The Author(s) 2011. This article is published with open access at Springerlink.com

\begin{abstract}
Aims/hypothesis Obesity is strongly associated with the development of non-alcoholic fatty liver disease (NAFLD). The cytokine osteopontin (OPN) was recently shown to be involved in obesity-induced adipose tissue inflammation and reduced insulin response. Accumulating evidence links OPN to the pathogenesis of NAFLD. Here we aimed to identify the role of OPN in obesity-associated hepatic steatosis and impaired hepatic glucose metabolism.

Methods Wild-type (WT) and Opn (also known as Spp1) knockout $\left(\mathrm{Oph}^{-/-}\right)$mice were fed a high-fat or low-fat diet to study OPN effects in obesity-driven hepatic alterations.
\end{abstract}

Electronic supplementary material The online version of this article (doi:10.1007/s00125-011-2170-0) contains supplementary material, which is available to authorised users.

F. W. Kiefer $\cdot$ B. Pfau $\cdot$ B. Legerer · A. Neuhofer $\cdot$ M. Zeyda

T. M. Stulnig $(\bowtie)$

Department of Medicine III,

Clinical Division of Endocrinology and Metabolism,

Medical University of Vienna,

Waehringer Guertel 18-20,

1090 Vienna, Austria

e-mail: thomas.stulnig@meduniwien.ac.at

S. Neschen $\cdot$ M. Kahle $\cdot$ M. Hrabé de Angelis

Institute of Experimental Genetics,

Helmholtz Zentrum Muenchen,

German Research Center for Environmental Health,

Neuherberg, Germany

M. Schlederer $\cdot$ M. Mair $\cdot$ L. Kenner

Ludwig Boltzmann Institute for Cancer Research,

Vienna, Austria

J. Plutzky

Department of Medicine, Brigham and Women's Hospital Boston,

Cardiovascular Division, Harvard Medical School,

Boston, MA, USA
Results We show that genetic OPN deficiency protected from obesity-induced hepatic steatosis, at least in part, by downregulating hepatic triacylglycerol synthesis. Conversely, absence of OPN promoted fat storage in adipose tissue thereby preventing the obesity-induced shift to ectopic fat accumulation in the liver. Euglycaemic-hyperinsulinaemic clamp studies revealed that insulin resistance and excess hepatic glucose production in obesity were significantly attenuated in $\mathrm{Opn}^{-1-}$ mice. OPN deficiency markedly improved hepatic insulin signalling as shown by enhanced insulin receptor substrate- 2 phosphorylation and prevented upregulation of the major hepatic transcription factor Forkhead box $\mathrm{O} 1$ and its gluconeogenic target genes. In addition, obesity-driven hepatic inflammation and macrophage accumulation was blocked by OPN deficiency. Conclusions/interpretation Our data strongly emphasise OPN as mediator of obesity-associated hepatic alterations including steatosis, inflammation, insulin resistance and excess gluconeogenesis. Targeting OPN action could therefore provide a novel therapeutic strategy to prevent obesity-related complications such as NAFLD and type 2 diabetes.

Keywords Gluconeogenesis · High-fat diet · Inflammation . Insulin resistance $\cdot$ Non-alcoholic fatty liver disease

$\begin{array}{ll}\text { Abbreviations } \\ \text { 2-[ }\left[{ }^{14} \mathrm{C}\right] \mathrm{DG} & \left.\text { 2-Deoxy-D-[1- }{ }^{14} \mathrm{C}\right] \text { glucose } \\ \text { ALT } & \text { Alanine aminotransferase } \\ \text { FOXO1 } & \text { Forkhead box O1 } \\ \text { GINF } & \text { Glucose infusion rate } \\ \text { GWAT } & \text { Gonadal white adipose tissue } \\ \text { HF } & \text { High-fat diet } \\ \text { ITT } & \text { Insulin tolerance test } \\ \text { LF } & \text { Low-fat diet }\end{array}$




$\begin{array}{ll}\text { MCP-1 } & \text { Monocyte chemoattractant protein-1 } \\ \text { NAFLD } & \text { Non-alcoholic fatty liver disease } \\ \text { NASH } & \text { Non-alcoholic steatohepatitis } \\ \text { NF-KB } & \text { Nuclear factor } \mathrm{kB} \\ \text { OPN } & \text { Osteopontin } \\ \text { PPAR } & \begin{array}{l}\text { Peroxisome proliferator-activated receptor } \\ \text { PGC }\end{array} \\ & \begin{array}{l}\text { Peroxisome proliferator-activated receptor } \\ \text { coactivator }\end{array} \\ \text { SAP } & \text { Serum amyloid P } \\ \text { SREBP-1c } & \text { Sterol regulatory element-binding protein 1 } \\ \text { SWAT } & \text { Subcutaneous white adipose tissue } \\ \text { WT } & \text { Wild-type }\end{array}$

\section{Introduction}

The obesity epidemic is tightly linked to a spectrum of hepatic disorders collectively known as non-alcoholic fatty liver disease (NAFLD). NAFLD has become an important public health issue because of its high prevalence and association with serious cardiometabolic abnormalities, including metabolic syndrome, type 2 diabetes and coronary heart disease [1, 2]. NAFLD spans a spectrum from simple hepatic steatosis through non-alcoholic steatohepatitis (NASH) ultimately leading to liver fibrosis and cirrhosis [3].

Increased energy intake exceeding energy dissipation promotes ectopic triacylglycerol storage in non-fat organs such as skeletal muscle and liver [4]. Triacylglycerol homeostasis in the liver is regulated by a complex interplay between hepatic plasma NEFA uptake and de novo fatty acid and triacylglycerol synthesis (lipogenesis) on the one hand, as well as fatty acid oxidation and triacylglycerol export by VLDLs on the other hand. Hepatic steatosis develops when the rate of hepatic fatty acid input (uptake and synthesis) exceeds the rate of fatty acid output (oxidation and secretion) [5].

Steatosis is associated with hepatic insulin resistance, i.e. the reduced sensitivity of the liver to the suppressive effects of insulin on glucose and VLDL triacylglycerol production. Decreased ability of insulin to suppress the hepatic output of glucose and VLDL contributes to hyperglycaemia and hyperlipidaemia, intrinsic features of the metabolic syndrome. As such, hepatic steatosis is regarded as the hepatic component of the metabolic syndrome [4]. Insulin resistance in liver, adipose tissue, and skeletal muscle is directly related to intrahepatic triacylglycerol content. However, it still remains a matter of debate whether insulin resistance is a cause or consequence of NAFLD [6,7].

In addition to lipid accumulation, another hallmark of NAFLD is hepatic inflammation resulting in NASH. Similar to its effects on adipose tissue, obesity induces inflammatory alterations in the liver as reflected by a higher abundance of immune cells such as macrophages [8], increased cytokine production [3] and by activation of the nuclear factor $\kappa B(\mathrm{NF}-\mathrm{kB})$ pathway [9]. Adipose tissue inflammation and unfavourable adipokine secretion are enhanced in individuals with NAFLD compared with individuals with normal intrahepatic triacylglycerol content [10], indicating that inflammatory factors released by the adipose tissue could be involved in the pathogenesis of NAFLD and hepatic insulin resistance [11]. Only recently liver macrophages were reported to be increased in number and to promote hepatic steatosis and insulin resistance in obesity [12]. Chemokines such as monocyte chemoattractant protein-1 (MCP-1) contribute to obesity-induced recruitment of macrophages to the liver $[12,13]$.

Osteopontin (OPN) is a multifunctional protein produced in numerous cells including activated macrophages and $\mathrm{T}$ cells, osteoclasts, smooth muscle, endothelial cells, and also hepatocytes $[14,15]$. OPN induces the production of a variety of proinflammatory cytokines and chemokines in peripheral blood mononuclear cells [16] and supports migration of monocytes/macrophages [15]. Systemic concentrations as well as adipose content of OPN are significantly elevated in obese patients and mice [17, 18]. It has recently been suggested that genetic OPN deficiency and antibody-mediated neutralisation improve obesity-associated adipose tissue inflammation and glucose tolerance [19, 20].

Notably, OPN content is also markedly upregulated in the liver in obesity, and hepatic OPN levels correlate with liver triacylglycerol content [18, 21-23]. Within the liver, OPN is predominantly produced in inflammatory cells but also hepatocytes [24]. A role for OPN in the pathogenesis of NAFLD was suggested in mice fed a methionine- and choline-deficient diet [22, 23]. Recently, antibody-mediated OPN neutralisation was shown to protect against high-fat diet-induced hepatic macrophage infiltration [19] and Dgalactosamine-induced inflammatory liver injury [24]. However, a functional role of OPN in obesity-associated hepatic steatosis and liver insulin sensitivity still remains unclear.

Here we show that genetic OPN deficiency prevents highfat diet-induced hepatic lipid accumulation and enhances hepatic insulin action to suppress gluconeogenesis. Obesitydriven liver inflammation, as well as deleterious signal transduction pathways related to hepatic insulin resistance, is attenuated in obese $\mathrm{Opn}$ (also known as $\operatorname{Spp} 1)^{-/-}$mice. Hence, targeting OPN action in vivo could provide a novel therapeutic strategy to combat obesity-associated hepatic steatosis and related metabolic complications.

\section{Methods}

Animals and diet $\mathrm{C} 57 \mathrm{BL} / 6 \mathrm{~J}$ wild-type (WT) and B6.Cg$\mathrm{Spp} 1^{\mathrm{tm} 1 \mathrm{Blh} / \mathrm{J}}$ (here referred to as $\mathrm{Opn}$ knockout $\left[\mathrm{Opn}^{-/-}\right]$) 
mice were purchased from Charles River Laboratories (Sulzfeld, Germany). At 7 weeks of age, male littermates were placed for 24 weeks on a high-fat (HF, $60 \%$ of energy from fat, D12492, Research Diets, New Brunswick, NJ, USA) and a low-fat diet (LF, $10 \%$ of energy from fat, D12450B, Research Diets) to induce obesity or to serve as lean controls, respectively. All mice were housed in a specific pathogen-free facility that maintained a $12 \mathrm{~h} \mathrm{light/}$ dark cycle. Mice had free access to food and water and food intake was monitored. The protocol was approved by the local ethics committee for animal studies and the Federal Ministry for Science and Research and followed the guidelines on accommodation and care of animals formulated by the European Convention for the Protection of Vertebrate Animals Used for Experimental and Other Scientific Purposes.

Insulin treatment in mice WT and $\mathrm{Opn}^{-1-}$ mice were kept on $\mathrm{HF}$ for 24 weeks. After $6 \mathrm{~h}$ fasting, mice were intraperitoneally injected with $1.5 \mathrm{U} / \mathrm{kg}$ insulin (Actrapid, Novo Nordisk, Bagsværd, Denmark) and saline, respectively, $15 \mathrm{~min}$ prior to being killed ( $n=5$ per group). The liver was removed and immediately snap frozen for later immunoblot analyses.

Metabolic measurements Plasma glucose, triacylglycerol, and NEFA concentrations were measured in EDTA plasma using an automated analyser (Falcor 350, A. Menarini Diagnostics, Florence, Italy). We used commercially available ELISA kits to determine plasma insulin (Mercodia AB, Uppsala, Sweden) and serum amyloid P (SAP) (Alpco Diagnostics, Windham, NH, USA). Plasma concentrations of alanine aminotransferase (ALT) were measured using the Reflotron analysis system (Roche, Mannheim, Germany). Insulin tolerance tests (ITTs) were performed after a $3 \mathrm{~h}$ fasting period. Blood glucose concentrations were measured before and 30,60, 90 and $120 \mathrm{~min}$ after an intraperitoneal injection of recombinant human insulin (Actrapid, Novo Nordisk, $0.75 \mathrm{U} / \mathrm{kg}$ body weight for the $\mathrm{HF}$ group and $0.25 \mathrm{U} / \mathrm{kg}$ for the LF group, respectively).

Euglycaemic-hyperinsulinaemic clamp studies WT and $\mathrm{Opn}^{-1-}$ mice were fed a HF diet for 24 weeks in order to induce obesity. One week prior to clamp experiments an intravenous silicone catheter was inserted into the right jugular vein of weight-matched mice ( $n=10$ per group). Mice were overnight fasted prior to the clamp procedure. In vivo experiments lasted for $240 \mathrm{~min}$ and consisted of a 120 min basal period followed by a 120 min euglycaemichyperinsulinaemic clamp as previously described $[25,26]$. Briefly, the clamp period was initiated by a $600 \mathrm{pmol} / \mathrm{kg}$, 3 min prime, followed by a $36 \mathrm{pmol} \mathrm{kg}^{-1} \mathrm{~min}^{-1}$ continuous infusion of insulin (Huminsulin Normal, Lilly Deutschland,
Bad Homburg, Germany) raising plasma insulin concentrations within a physiological range. By means of a variable $20 \%$ glucose infusion 'steady state' (minute 90 120) conditions for plasma glucose concentration and specific activity were achieved. At minute 70 , a single intravenous 2-deoxy-D-[1- $\left.{ }^{14} \mathrm{C}\right]$ glucose $\left(2-\left[{ }^{14} \mathrm{C}\right] \mathrm{DG}\right.$, $370 \mathrm{kBq}$ ) injection was administered. Plasma samples for determination of insulin-stimulated plasma $\left[{ }^{3} \mathrm{H}_{3}\right]$ glucose, ${ }^{3} \mathrm{H}_{2} \mathrm{O}$ and 2- $\left[{ }^{14} \mathrm{C}\right]$ DG concentrations were collected at $t=$ $77.5,80,85,90,100,110$, and $120 \mathrm{~min}$ of the glucose clamp, and basal $\left[{ }^{3} \mathrm{H}_{3}\right]$ glucose concentrations in the final $10 \mathrm{~min}$ of the basal period. At the end of each experiment, organs (liver, gonadal white adipose tissue [GWAT], and M. gastrocnemius) were immediately dissected, and snap frozen.

Tissue $2-\left[{ }^{14} C\right] D G$ uptake $2-\left[{ }^{14} \mathrm{C}\right] \mathrm{DG}$ injected during the steady state conditions of the euglycaemic-hyperinsulinaemic clamp experiments resulted in intracellular accumulation of $2-\left[{ }^{14} \mathrm{C}\right] \mathrm{DG}-6$-phosphate, which was separated from $2-\left[{ }^{14} \mathrm{C}\right] \mathrm{DG}$ using ion-exchange columns (Bio-Rad, Hercules, CA, USA). Tissue 2-[ $\left[{ }^{14} \mathrm{C}\right] \mathrm{DG}$ uptake was calculated from the plasma 2-[ $\left.{ }^{14} \mathrm{C}\right] \mathrm{DG}$ AUC and tissue 2-[ $\left.{ }^{14} \mathrm{C}\right] \mathrm{DG}-6$ phosphate content as previously described [26].

Determination of liver triacylglycerol content Liver triacylglycerol was determined following lipid extraction as described previously [27], modified by using a commercially available enzymatic reagent (A. Menarini Diagnostics).

Histology and immunohistochemistry Liver samples were fixed with neutral buffered $4 \%$ paraformaldehyde and subsequently paraffin embedded. Haematoxylin-eosin staining was performed in liver as described [22]. NAFLD activity scores were determined by a certified pathologist blinded to mouse genotype and dietary intervention. After dewaxing and rehydration, immunohistochemical staining for MAC-2 (Serotec, Oxford, UK) was performed using the $\mathrm{ABC}$ kit (Vector Laboratories, Burlingame, CA, USA) according to the manufacturer's recommendations. As a negative control, staining was performed on selected sections with isotype control. Samples were analysed with standard light microscopy.

Immunoprecipitation and immunoblotting Standard immunoblotting techniques were applied as described previously [28] using specific antibodies against forkhead box O1 (FOXO1; Santa Cruz Biotechnology, Santa Cruz, CA, USA), thymoma viral proto-oncogene 1 (AKT) and pAKT (ser473) (Cell Signaling, Danvers, MA, USA). IRS-2 was immunoprecipitated from liver tissue extracts (anti-IRS-2 polyclonal antibody, Cell Signaling). IRS-2 tyrosine phosphorylation was determined using anti-phosphotyrosine 
mAb (4G10) horse radish peroxidase (HRP) conjugate (Upstate, Lake Placid, NY, USA).

Reverse transcription and gene expression Adipose tissue and liver was homogenised in TRIzol reagent (Invitrogen, Carlsbad, CA, USA) and RNA was isolated and transcribed to cDNA according to the manufacturer's protocol [19]. Gene expression normalised to Ubiquitin $\mathrm{C}$ was analysed by quantitative real-time RT-PCR on an ABI Prism 7000 cycler using commercial Assays-on-Demand kits (all Applied Biosystems, Foster City, CA, USA). Gene expression was compared with WT HF, which was considered to be $100 \%$.

Statistics All data are given as means \pm SE. All data were normally distributed by visual inspection. Comparisons between genotypes on the same diet were assessed by unpaired two-tail Student's $t$ tests. Multiple regression analysis was performed to evaluate association between liver and GWAT weight in WT and $O p n^{-1-}$ mice. A $p$ value of 0.05 or less was considered to be statistically significant.

\section{Results}

OPN deficiency protects from obesity-induced hepatic steatosis To assess the role of OPN in obesity-associated NAFLD and hepatic insulin resistance, WT and $\mathrm{Opn}^{-1-}$ mice were fed either the HF or LF diet for 24 weeks to induce obesity or to serve as lean controls, respectively. Whereas OPN deficiency did not affect body weight gain or feed efficiency, liver weight was significantly lower in obese $\mathrm{Opn}^{-1-}$ mice compared with obese WT mice (Table 1). Systemic concentrations of glucose, triacylglycerols and NEFA did not differ between the two genotypes, while the obesity-induced rise in circulating plasma insulin was drastically blunted in $\mathrm{Opn}^{-/-}$mice to one third of the levels measured in WT mice. ALT, a marker of hepatocyte damage, was similarly reduced in obese $\mathrm{Opn}^{-1-}$ mice (Table 1). HF feeding induced severe lipid accumulation in livers of WT mice as determined by liver histology and quantification of hepatic triacylglycerol content. Strikingly, $\mathrm{Opn}^{-/-}$mice on HF were markedly protected from dietinduced hepatic steatosis (Fig. 1)

OPN deficiency counteracts hepatic insulin resistance and excessive glucose production We examined whether lack of OPN affects obesity-associated hepatic insulin resistance and gluconeogenesis. First we performed ITTs in WT and $\mathrm{Opn}^{-1-}$ mice showing that the insulin effect on plasma glucose was comparable in lean mice irrespective of the genotype. Notably, glucose clearance following insulin injection in obese HF-fed animals was significantly accelerated in $\mathrm{Opn}^{-/-}$mice compared with WT controls, indicating improved whole-body insulin sensitivity (Fig. 2a, b). In order to directly evaluate insulin sensitivity and the relative contribution of the liver to this metabolic phenotype we performed euglycaemic-hyperinsulinaemic clamp tests in obese WT and $\mathrm{Opn}^{-1-}$ mice. An increased glucose infusion rate (GINF) was required in obese OPN deficient mice to maintain euglycaemia during the clamp, confirming improved whole-body insulin sensitivity compared with WT controls (Fig. 2c). Basal glucose production was unchanged between both genotypes but, importantly, insulin-mediated suppression of hepatic glucose production was significantly enhanced in obese $\mathrm{Opn}^{-1-}$ mice compared with WT animals (Fig. 2d, e). Whole-body glycolytic activity under insulin-stimulated conditions was unaffected by OPN deficiency (Fig. 2f). To determine if organs other than liver contributed to the

Table 1 Body characteristics and plasma variables

\begin{tabular}{|c|c|c|c|c|}
\hline Variable & WT LF & $O p n^{-/-} \mathrm{LF}$ & WT HF & $\mathrm{Opn}^{-/-} \mathrm{HF}$ \\
\hline Body mass (g) & $32.5 \pm 1.0$ & $30.7 \pm 0.7$ & $50.8 \pm 0.6$ & $50.6 \pm 0.9$ \\
\hline Liver mass (g) & $1.34 \pm 0.07$ & $1.41 \pm 0.06$ & $2.08 \pm 0.11$ & $1.76 \pm 0.10^{*}$ \\
\hline Feed efficiency & $2.08 \pm 0.41$ & $1.42 \pm 0.45$ & $8.35 \pm 1.24$ & $8.22 \pm 1.92$ \\
\hline Glucose $(\mathrm{mmol} / \mathrm{l})$ & $7.50 \pm 0.06$ & $6.92 \pm 0.69$ & $15.15 \pm 0.88$ & $14.77 \pm 0.76$ \\
\hline Insulin (pmol/1) & $55.8 \pm 8.0$ & $40.5 \pm 4.3$ & $448.2 \pm 65.9$ & $134.7 \pm 24.9 * * *$ \\
\hline Triacylglycerol (mmol/l) & $0.41 \pm 0.03$ & $0.38 \pm 0.01$ & $0.64 \pm 0.06$ & $0.67 \pm 0.09$ \\
\hline NEFA $(\mu \mathrm{mol} / \mathrm{l})$ & $224.5 \pm 13.2$ & $271.3 \pm 27.0$ & $328.7 \pm 26.2$ & $286.3 \pm 31.9$ \\
\hline ALT (U/1) & $15.1 \pm 1.6$ & $11.0 \pm 2.1$ & $53.9 \pm 6.9$ & $31.6 \pm 4.3 * *$ \\
\hline Serum amyloid $\mathrm{P}(\mathrm{ng} / \mathrm{ml})$ & $32.7 \pm 5.8$ & $34.6 \pm 6.1$ & $72.9 \pm 10.9$ & $40.6 \pm 8.7^{*}$ \\
\hline
\end{tabular}

Data are expressed as mean \pm SEM

WT and $\mathrm{Opn}^{-/-}$mice were fed LF or HF for 24 weeks ( $n=10$ per group). Body and liver mass were measured. Feed efficiency was determined as weight gain per food consumption. Blood samples were obtained after $3 \mathrm{~h}$ fasting period and analysed for depicted plasma variables

Significant differences between genotypes on the same diet are indicated as ${ }^{*} p \leq 0.05,{ }^{* *} p \leq 0.01,{ }^{* * *} p \leq 0.001$ 
a
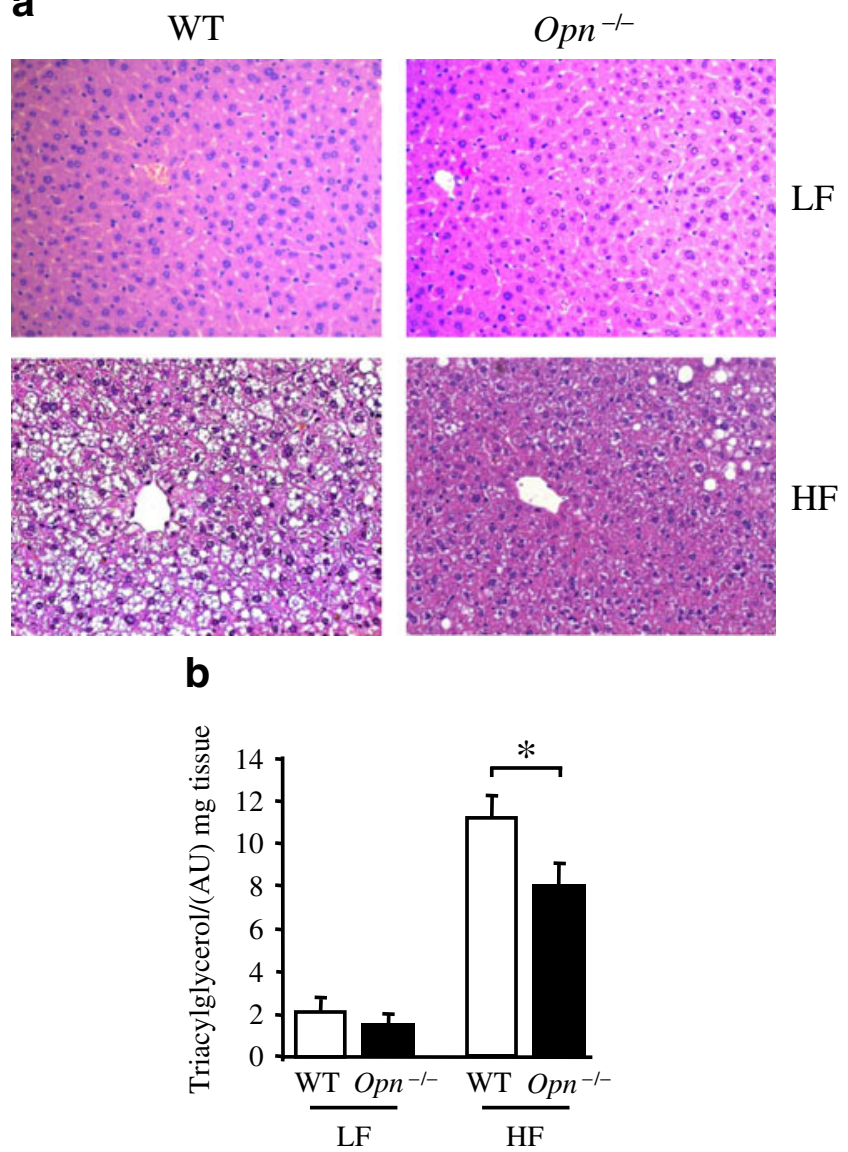

Fig. 1 OPN deficiency protects against obesity-induced hepatic steatosis. WT and $\mathrm{Opn}^{-/-}$mice were kept either on LF or HF diet for 24 weeks ( $n=10$ per group). a Haematoxylin-eosin staining on liver paraffin sections. Representative pictures are given in 20-fold magnification. b Hepatic triacylglycerol content was determined in lipid extraction by an enzymatic test. ${ }^{*} p \leq 0.05$ between genotypes. AU, arbitrary unit

metabolic improvement we measured insulin-stimulated $2-\left[{ }^{14} \mathrm{C}\right] \mathrm{DG}$ uptake both in GWAT and skeletal muscle, without detecting a difference between WT and $\mathrm{Opn}^{-1-}$ mice (Fig. $2 \mathrm{~g}, \mathrm{~h}$ ). Hence, attenuated hepatic steatosis in obese OPN deficient mice is paralleled by improved hepatic insulin sensitivity and restored insulin-mediated suppression of hepatic glucose production.

Reduced hepatic triacylglycerol synthesis may contribute to the absence of hepatic steatosis in OPN deficiency In order to investigate possible mechanisms underlying the prevention of hepatic steatosis in OPN deficient mice, we analysed the expression of genes relevant for fatty acid and triacylglycerol homeostasis in the liver. Markers of fatty acid synthesis such as Fasn and Acaca did not differ between obese WT and $\mathrm{Opn}^{-1-}$ mice (Fig. 3a). Neither was enhanced fatty acid oxidation likely to contribute to reduced hepatic steatosis since important transcription factors driving $\beta$-oxidation were unchanged or even downregulated as shown for Ppara and Ppargcla, respectively (Fig. 3a). However, gene expression of the major transcriptional regulator of hepatic lipogenesis Srebfl was markedly decreased in $\mathrm{Opn}^{-1-}$ compared with WT animals (Fig. 3b). This was similarly found to be the case for Pparg (Fig. 3c), a hepatic sterol regulatory element-binding protein 1 (SREBP-1c) target gene [29]. Given the downregulation of hepatic Pparg mRNA expression, we next examined its transcriptional targets Dgat1 and -2, which catalyse the formation of triacylglycerols from diacylglycerol and acylcoenzyme A [30]. Notably, Dgat1 expression was significantly downregulated in obese $\mathrm{Opn}^{-1-}$ livers while Dgat2 followed the same trend, although the change was not significant (Fig. 3d, e). These results strongly emphasise that reduced hepatic triacylglycerol synthesis by downregulation of Dgat expression could contribute to the absence of hepatic steatosis in obese OPN-deficient mice.

Lack of OPN favours lipid accumulation in visceral fat instead of liver Decreased hepatic triacylglycerol content in obese $\mathrm{Opn}^{-/-}$mice (Fig. 1) indicates the prevention of ectopic fat accumulation, suggesting orthotopic fat storage in adipose tissue. Subcutaneous white adipose tissue (SWAT) weight was similar in both genotypes on the same diet, whereas GWAT weight was strikingly increased in $\mathrm{Opn}^{-/-}$compared with WT animals, both on HF diets (Fig. 4a, b). Since liver weight and steatosis were significantly reduced in obese $\mathrm{Opn}^{-/-}$vs WT mice (Table 1 and Fig. 1), we next investigated a possible relationship between liver and GWAT mass. Multiple regression analyses revealed no association between liver and GWAT mass in lean animals $\left(r^{2}=0.05, p=0.28\right.$, Fig. $\left.4 c\right)$. Notably, liver mass was strongly negatively associated with GWAT weight in obese WT and $O p n^{-1-}$ mice $\left(r^{2}=0.42, p=0.008\right.$, Fig. $4 d$ ), indicating that storage of excess lipids in gonadal adipose tissue prevents hepatic steatosis in the absence of OPN. Taken together, OPN deficiency inhibits ectopic fat deposition in liver thereby protecting from deleterious effects on hepatic metabolism.

Genetic OPN deletion improves hepatic insulin signalling in obesity and regulates gluconeogenic enzyme expression via FOXO1 HF-induced obesity impairs insulin-stimulated tyrosine phosphorylation of IRSs, thereby causing insulin resistance [31]. Since IRS-2 is the predominant IRS in liver, we studied insulin-stimulated hepatic IRS-2 tyrosine phosphorylation in obese WT and $\mathrm{Opn}^{-/-}$mice, $15 \mathrm{~min}$ following intraperitoneal injection of $1.5 \mathrm{U} / \mathrm{kg}$ insulin or saline. Probably as a consequence of markedly elevated basal plasma insulin levels in WT mice, hepatic IRS-2 phosphorylation was pronounced under unstimulated conditions, but exogenous insulin failed to further stimulate 
a

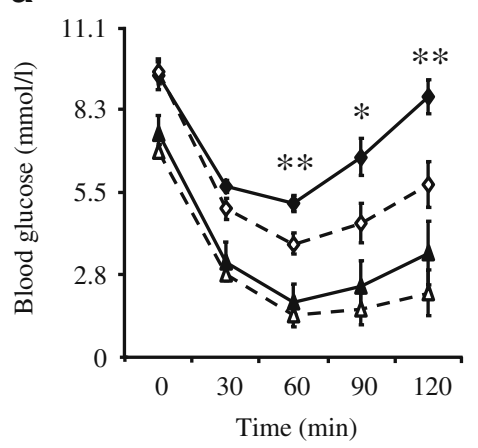

b

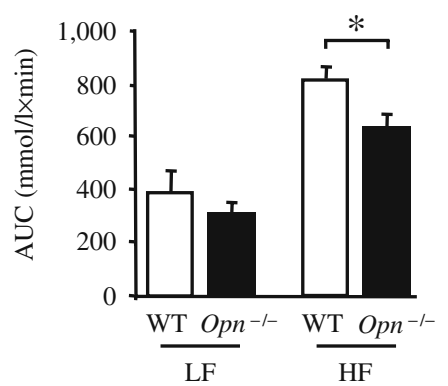

C

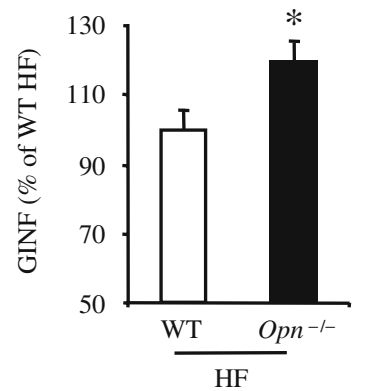

d

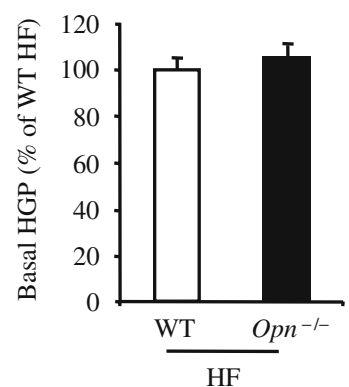

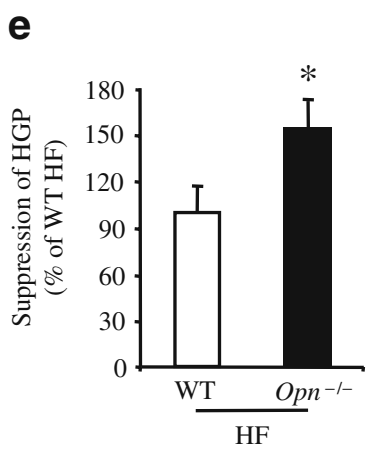

f

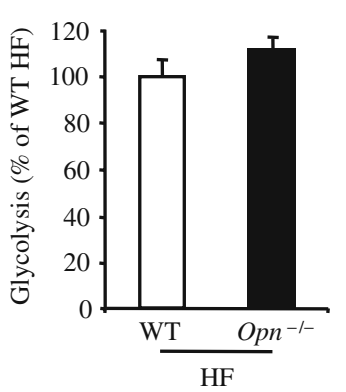

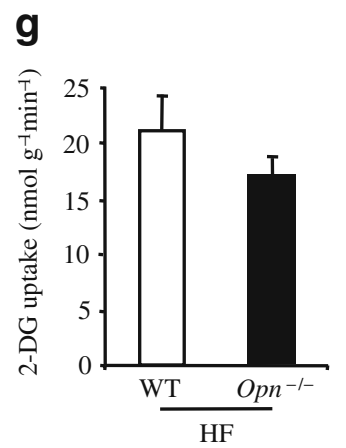

h

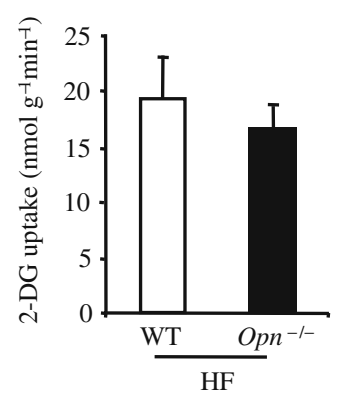

Fig. 2 OPN deficiency reduces obesity-induced hepatic insulin resistance and glucose production. WT and $\mathrm{Opn}^{-/-}$mice were kept either on LF or HF for 24 weeks. a, b An ITT was performed in WT (solid lines) and $\mathrm{Opn}^{-/-}$mice (dashed lines) after feeding LF (triangles) or HF (squares) ( $n=10$ per group). Glucose concentrations during ITT (a) and area under the curve are given (b). ch Euglycaemic-hyperinsulinaemic clamp studies were conducted in

IRS-2 phosphorylation (Fig. 5a). Conversely, tyrosinephosphorylated IRS-2 was barely detectable at baseline in HF-fed OPN deficient mice but significantly increased following insulin stimulation (Fig. 5a). In addition, insulinstimulated serine phosphorylation of AKT, which mediates signal transduction downstream of IRS, was markedly more enhanced in $\mathrm{Opn}^{-1-}$ vs WT mice (Fig. 5b). These data strongly indicate that impaired hepatic insulin signal transduction in obesity is improved in the absence of OPN. Intact insulin signalling is required for the inactivation of the transcription factor Foxol [32], a potent regulator of hepatic gluconeogenesis. Improved insulin signalling in obese $\mathrm{Opn}^{-/-}$livers, together with a downregulation of peroxisome proliferator-activated receptor gamma coactivator (PGC)-1 $\alpha$ (Fig. 3a), a transcriptional coactivator of FOXO1 [33], prompted us to examine hepatic abundance of FOXO1 in WT and OPN-deficient mice. Foxol mRNA and protein levels were significantly enhanced in obese WT livers, whereas absence of OPN protected from HF-induced hepatic FOXO1 upregulation (Fig. 5c, d). In addition, expression of the FOXO1 targets G6pc and Mttp was upregulated in obese WT livers but remained close to lean levels in OPN-deficient mice mice kept on HF ( $n=10$ per group). GINF (c) was measured during steady state conditions as well as basal hepatic glucose production (HGP; d), insulin-mediated suppression of HGP (e) and whole-body glycolysis (f). $\mathbf{g}, \mathbf{h}$ Tissue-specific glucose uptake was analysed by enrichment of phosphorylated 2-[ $\left[{ }^{14} \mathrm{C}\right] \mathrm{DG}$ in $\mathrm{M}$. gastrocnemius (g) and GWAT (h). ${ }^{*} p \leq 0.05 ; * * p \leq 0.01$

(Fig. 5e, f). Thus, downregulation of hepatic FOXO1 and gluconeogenic enzyme production probably contributes to the beneficial effects of OPN deficiency on glucose and lipid homeostasis in obesity.

\section{OPN deficiency prevents obesity-mediated hepatic inflammation} and macrophage accumulation In order to assess NAFLD disease stage, liver histology was performed and the NAFLD activity score was determined by a certified pathologist. Mice on a normal chow diet did not show any alterations in liver histology irrespective of the genotype (electronic supplementary material [ESM] Fig. 1). However, 24 weeks of HF feeding significantly enhanced hepatic steatosis (Fig. 1), lobular inflammation and hepatocyte ballooning in WT but not in $\mathrm{Opn}^{-1-}$ mice (Fig. 6a, b). In addition, circulating concentrations of the liver-derived inflammatory marker serum amyloid P (SAP) were highly elevated in the plasma of obese WT, compared with OPN deficient, animals (Table 1). Increasing evidence links macrophage accumulation in the liver to hepatic steatosis and insulin resistance [12, 34]. OPN was recently shown to mediate obesity-induced adipose tissue macrophage accumulation $(19 ; 20)$. HF feeding raised gene 
a

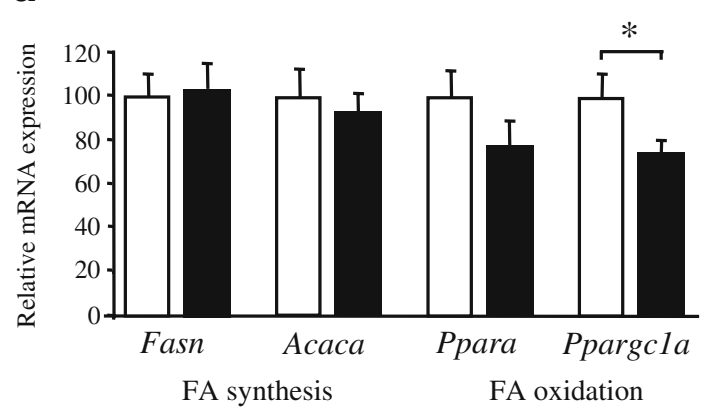

b

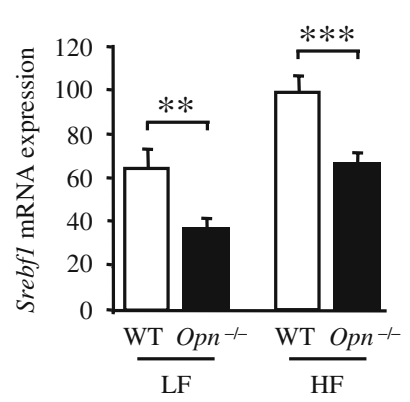

d

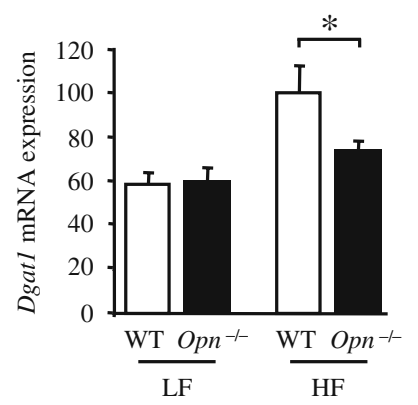

C

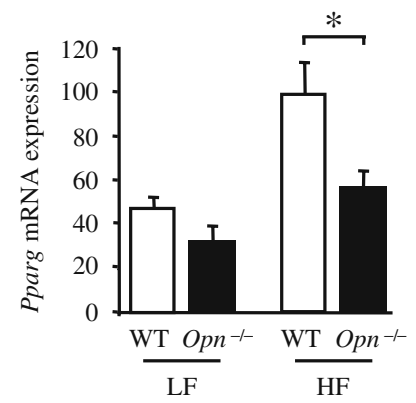

e

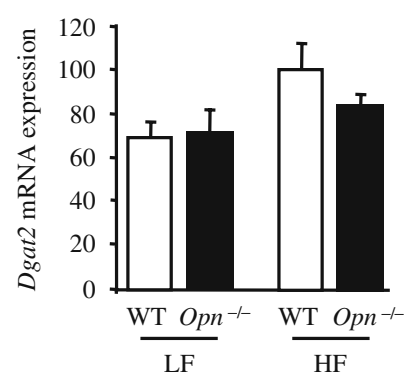

Fig. 3 OPN deficiency interferes with expression of lipogenic markers. WT and $\mathrm{Opn}^{-1-}$ mice were kept either on LF or HF for 24 weeks ( $n=10$ per group). Hepatic mRNA expression of Fasn and Acaca and the FA oxidation markers Ppara and Ppargcla (a); white bars: WT HF, black bars: $\mathrm{Opn}^{-/-} \mathrm{HF}$; as well as of transcription factors regulating lipid metabolism (Srebfl and Pparg; b, c) and enzymes controlling triacylglycerol synthesis (Dgat1 and -2 ; d, e) was analysed. $* p \leq 0.05, * * p \leq 0.01, * * * p \leq 0.001$. Gene expression is shown as percentages relative to WT HF

expression of the macrophage marker Emrl significantly less in OPN-deficient than in WT livers (Fig. 6c). Hepatic gene expression of $\mathrm{Ccl} 2$ was markedly increased in obesity in WT but not in $\mathrm{Opn}^{-/-}$animals (Fig. 6d). Immunohistochemical analysis of liver sections revealed a sparse number of macrophages in lean mice without any genotypic difference (not shown). In contrast, hepatic macrophages were highly abundant in obese WT mice and were clustered in close vicinity to lipid droplets (Fig. 6e) resembling the so-called crown-like structures described in obese adipose tissue [35]. However, only a few such structures were found

in livers of obese $\mathrm{Opn}^{-/-}$mice, where macrophages were less abundant and located rather individually throughout the tissue (Fig. 6e). Macrophages are the major source of obesity-driven cytokine production not only in adipose tissue but also in liver [36]. Hepatic mRNA expression of inflammatory $\operatorname{Tnf}$ and $\operatorname{Tg} f b 1$, known to be crucially involved in pathogenesis and progression of NAFLD [37, 38] was increased with HF in WT mice but significantly less in $\mathrm{Opn}^{-/-}$mice (Fig. 6f, g). Taken together, these results reveal that OPN is not only a mediator of hepatic steatosis but also of obesity-driven inflammatory alterations including macrophage accumulation in the liver.

\section{Discussion}

The growing prevalence of obesity and related inflammatory and metabolic disorders such as NAFLD and type 2 diabetes demands novel therapeutic approaches. Osteopontin is an inflammatory cytokine, hepatic content of which is upregulated in the course of human and murine hepatic steatosis and various models of liver injury [18, 21, 39]. Indirect evidence suggests that OPN is involved in hepatic steatosis, but a functional role has not been demonstrated. Here we show that genetic OPN deficiency prevents high- a

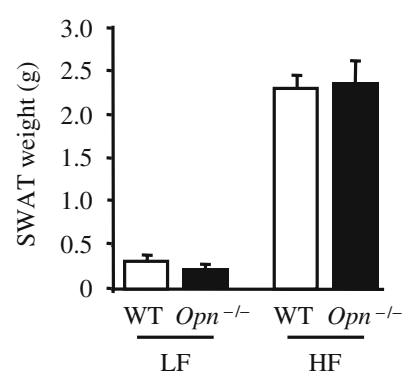

C

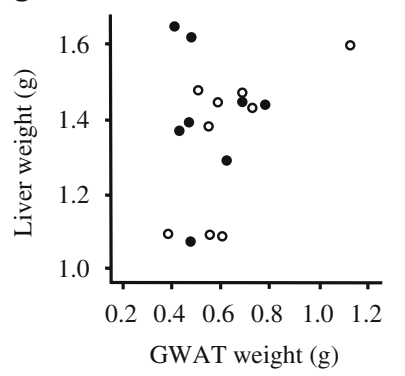

b

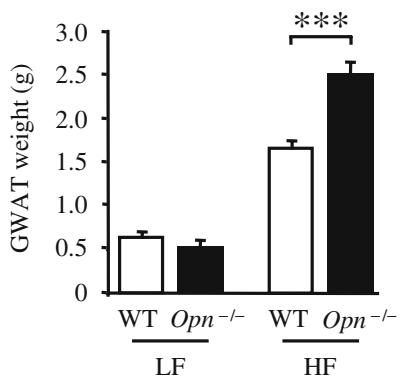

d

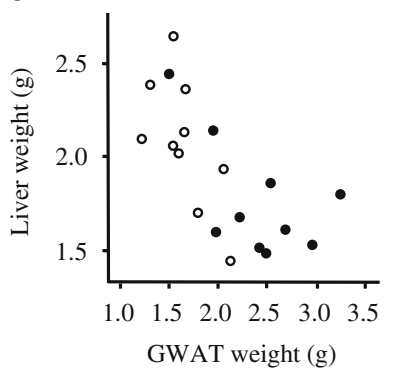

Fig. 4 Lack of OPN prevents hepatic lipid accumulation by orthotopic storage in GWAT. WT and $\mathrm{Opn}^{-/-}$mice were kept either on LF or HF for 24 weeks ( $n=10$ per group). SWAT (a) and GWAT (b) mass was measured immediately after dissection. Multiple regression analyses were performed between liver and GWAT mass of lean $\left(r^{2}=0.05, p=0.28 ; \mathbf{c}\right)$ and obese $\left(r^{2}=0.42, p=0.008 ; \mathbf{d}\right)$. WT, white circles; $\mathrm{Opn}^{-/-}$, black circles. ${ }^{* * *} p \leq 0.001$ 
a

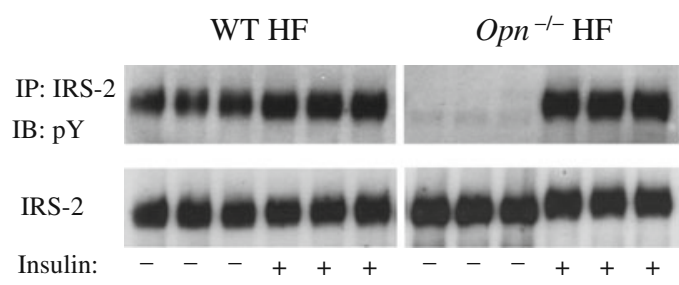

b

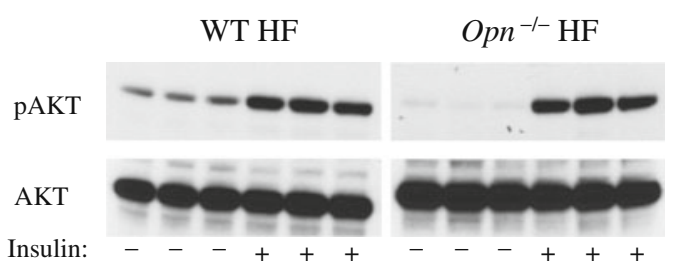

C

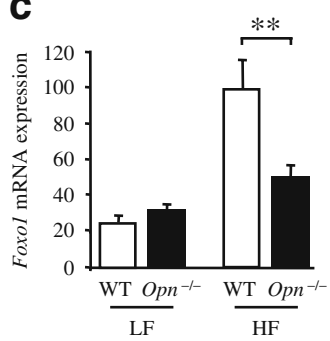

d

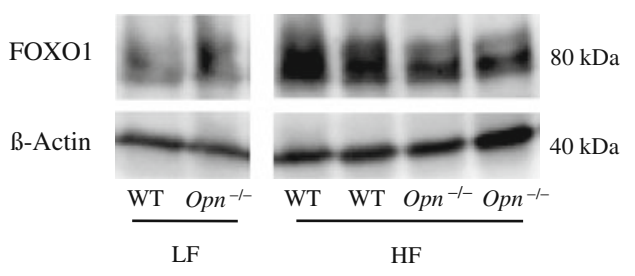

e

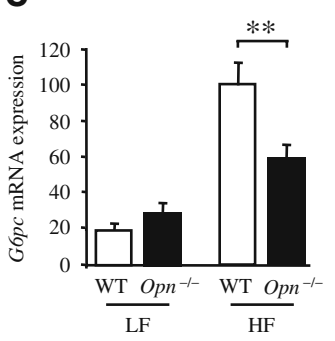

f

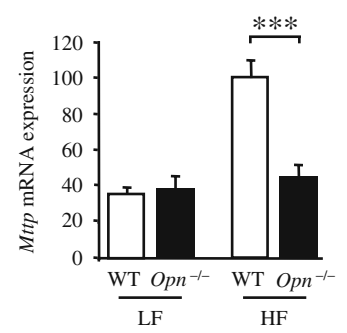

Fig. 5 OPN deficiency ameliorates hepatic insulin signalling and determines FOXO1 gluconeogenic enzyme levels in obesity. WT and $\mathrm{Opn}^{-1-}$ mice were kept on HF for 24 weeks. 15 min prior to sacrifice mice were intraperitoneally injected either with insulin $(1.5 \mathrm{U} / \mathrm{kg}$ ) or saline ( $n=5$ per group). IRS-2 tyrosine phosphorylation (pY) was analysed in liver tissue extracts following immunoprecipitation (IP) of IRS-2. Representative immunoblots (IB) are given (a). Phosphorylation (ser473) of AKT was determined in livers of obese
WT and $\mathrm{Opn}^{-1-}$ mice after intraperitoneal insulin injection. Representative immunoblots are given (b). Hepatic mRNA (c) and protein (d) levels of FOXO1 were determined in lean and obese WT and $\mathrm{Opn}^{-1-}$ mice ( $n=10$ per group). A representative blot is given. e, f mRNA expression of FOXO1 transcriptional targets G6pc (e) and Mttp (f) were analysed in livers of lean and obese WT and $\mathrm{Opn}^{-/-}$mice. ${ }^{* *} p \leq$ $0.01, * * * p \leq 0.001$. Gene expression is shown as percentages relative to WT HF fat diet-induced hepatic steatosis and inflammation, and markedly improves insulin signalling and insulin sensitivity in the liver, thereby improving hepatic glucose and lipid homeostasis. Thus, our data indicate that OPN is causally linked to obesity-induced detrimental hepatic alterations.

We and others have recently reported that hepatic OPN production is significantly upregulated in murine HFinduced and genetic obesity $[18,23]$ and correlates with the severity of hepatic steatosis in humans [21]. Here we demonstrate that $\mathrm{Opn}^{-1-}$ mice are protected from obesityinduced hepatic steatosis (Fig. 1). Hepatic steatosis is characterised by an imbalance between fatty acid input and output [5]. Expression of marker genes indicated unchanged fatty acid oxidation and synthesis between WT and $\mathrm{Opn}^{-/-}$livers in obesity despite decreased SREPB-1c levels in obese OPN-deficient mice (Fig. 3a, b). Interestingly, HF feeding upregulated hepatic production of the lipogenic factor Pparg in WT but not in $\mathrm{Opn}^{-/-}$, which could be the result of decreased SREBP-1c production and/ or the lack of OPN (Fig. 3c). Consequently, the expression of Dgat1, which is regulated by peroxisome proliferatoractivated receptor (PPAR)- $\gamma$ [29] and catalyses triacylglycerol formation [30], was significantly decreased (Fig. 3d). Hence, our data point toward unaltered transcriptional regulation of fatty acid metabolism, but reduced hepatic triacylglycerol synthesis, underlying the lack of steatosis in OPN deficiency. Given that NEFA concentrations as well as feed efficiency (Table 1), respiratory quotient and energy expenditure (data not shown and [20]) were unchanged between both genotypes, increased fatty acid dissipation was unlikely to occur in $\mathrm{Opn}^{-/-}$mice. Thus, we hypothesised that, in the absence of OPN, plasma NEFA were preferentially distributed toward non-hepatic tissue. Indeed, GWAT weight was strikingly increased in obese $\mathrm{Opn}^{-/-}$ mice (Fig. 4b) indicating that OPN deficiency facilitates orthotopic fat storage in adipose tissue thereby preventing its ectopic accumulation in the liver. This conclusion was corroborated by multiple regression analyses revealing a strong negative association between liver and GWAT weight in obese mice from both genotypes (Fig. 4d).

Visceral fat is metabolically highly active, in contrast to SWAT. The enlargement of GWAT, which is regarded as the visceral fat depot in mice, is frequently associated with insulin resistance [40]. We assume that the reduction of hepatic lipid content occurring in OPN deficient animals (Fig. 1) is more relevant for insulin sensitivity than the accompanying increase in GWAT mass (Fig 4b). Observations of decreased inflammatory alterations in GWAT of obese $\mathrm{Opn}^{-1-}$ mice [20] could contribute to the mitigation of metabolic perturbation occurring with GWAT enlargement in the absence of OPN.

Notably, decreased steatosis in obese $\mathrm{Opn}^{-1-}$ mice was paralleled by improved whole-body insulin sensitivity, which was mainly due to reduction in hepatic insulin resistance and glucose production as revealed by euglycaemic-hyperinsulinaemic clamp studies (Fig. 2). Enhanced 
a

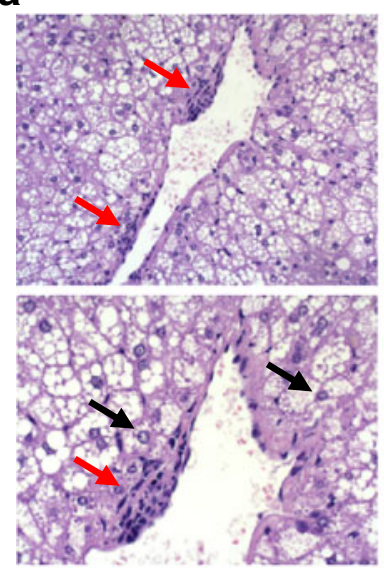

WT HF

b


C

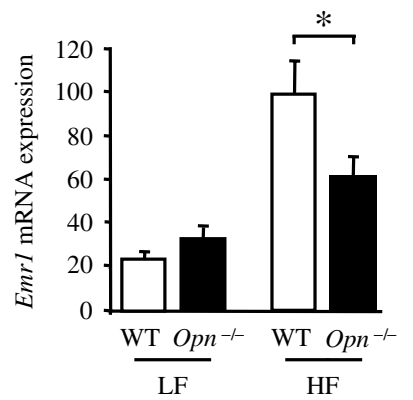

d

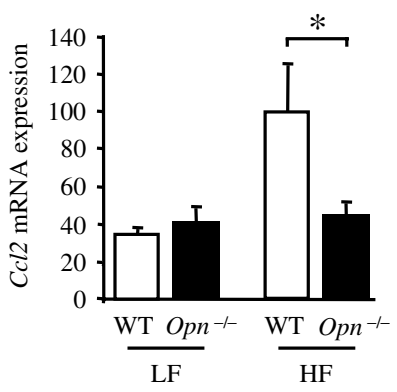

Fig. 6 Obesity-induced hepatic inflammation and macrophage accumulation is antagonised by OPN deficiency. WT and $\mathrm{Opn}^{-/-}$mice were kept either on LF or HF for 24 weeks ( $n=10$ per group). a Representative haematoxylin-eosin staining of liver paraffin sections is given. Black arrows indicate hepatocyte ballooning, red arrows point to portal leucocyte infiltrate. b NAFLD activity score (NAS) was determined by a certified pathologist. White bars, WT HF; black bars $\mathrm{Opn}^{-/-}$HF. c, d Hepatic mRNA expression of the macrophage marker Emrl (c) and the monocyte recruiting chemokine $C c l 2$ (d) was measured. e Macrophages were stained brown by immunohistochemistry using MAC-2 monoclonal antibody on paraffin sections of livers isolated from HF-fed mice. Representative pictures are given. $\mathbf{f}, \mathbf{g}$ mRNA expression of the inflammatory markers $\operatorname{Tn} f(\mathbf{f})$ and $T g f b l$ (g) was analysed in livers of WT and $\mathrm{Opn}^{-/-}$mice. ${ }^{*} p \leq 0.05,{ }^{* *} p \leq 0.01,{ }^{* * *} p \leq 0.001$. Gene expression is shown as percentages relative to WT HF

insulin-stimulated phosphorylation of IRS-2 and AKT in OPN deficient, compared with WT, livers provided additional evidence for ameliorated hepatic insulin signalling
(Fig. 5). A hallmark of hepatic insulin action is reduction of gluconeogenesis, which is downstream regulated by the transcription factor FOXO1 and its coactivator PGC- $1 \alpha$. Both FOXO1 and PGC- $1 \alpha$ were downregulated in livers from obese $\mathrm{Opn}^{-1-}$ compared with WT mice, as were the FOXO1 target genes G6pc and Mttp (Fig. 3a and Fig. 5) indicating abated gluconeogenesis. These results are in line with our previous observation that antibody-mediated OPN neutralisation reduces hepatic gluconeogenic enzyme production in HF-fed WT mice [19].

An obvious parallel between obesity-related pathologies of adipose tissue and liver pertains to the emerging role of inflammation. Evidence is growing that hepatic macrophages such as Kupffer cells are critically implicated in the pathogenesis and progression of NAFLD [34]. Only recently, chemical depletion of Kupffer cells was shown to prevent HF-induced hepatic steatosis and insulin resistance $[12,41,42]$. Here we demonstrate that OPN deficiency markedly counteracts obesity-induced hepatic inflammation and macrophage accumulation as assessed by NAFLD activity score, SAP concentrations and expression of macrophage markers, respectively (Fig. 6, Table 1). These data concur with recent reports showing that genetic OPN deletion and antibody-mediated OPN neutralisation antagonise macrophage recruitment in obese adipose tissue $[19,20]$. Macrophages are the major source of obesityassociated inflammatory cytokine production in liver as shown for adipose tissue $[36,40]$. OPN deficiency inhibited HF-induced upregulation of hepatic $T n f$ and $T g f b l$ gene expression (Fig. 6f, g), both of which are predominantly derived from hepatic macrophages and were repeatedly reported to underlie NAFLD progression [37, 38]. Lack of OPN also downregulated hepatic gene expression of MCP-1 (Fig. 6d), a chemokine that promotes hepatic macrophage infiltration and steatosis [13,43]. Since OPN and MCP-1 cooperate in monocyte chemotaxis [15, 44], abrogation of inflammatory cell migration is likely to account for reduced abundance of macrophages in livers from HF-fed $\mathrm{Opn}^{-/-}$ mice. Moreover, deficiency and neutralisation of OPN improved liver injury and fibrosis elicited by methionineand choline-deficient diet, concanavalin A, and D-galactosamine $[22,24,45,46]$ indicating a general implication of OPN in inflammatory liver damage.

In conclusion, at least two mechanisms are likely to contribute to the improvement of obesity-related hepatic steatosis and metabolic perturbation during OPN deficiency. On the one hand lack of OPN reduces hepatic triacylglycerol accumulation and fosters lipid storage in adipose tissue. On the other hand, hepatic inflammation is attenuated in the absence of OPN. Hence, OPN is shown here as a novel mediator of obesity-associated hepatic inflammation, steatosis and insulin resistance. Blocking OPN effects in vivo could normalise altered hepatic lipid 
and glucose homeostasis in obesity and therefore prevent progression of NAFLD and development of type 2 diabetes.

Acknowledgements We thank H. Schachner (Department of Pathology, Medical University of Vienna), L-I Ionasz (Clinical Division of Endocrinology and Metabolism, Department of Medicine III, Medical University of Vienna, Austria) N. Boche (Institute of Experimental Genetics, Helmholtz Zentrum Muenchen, German Research Center for Environmental Health) and the German Mouse Clinic, for excellent technical expertise. This work was supported by the Austrian Science Fund (project no. P18776-B11 and as part of the CCHD doctoral programme W1205-B09), the European Community's 7th Framework Programme (FP7/2007-2013) under grant agreement no. 201608 (all to T.M. Stulnig), and the National Genome Research Network of the German Federal Ministry of Education and Research (BMBF, NGFNplus grant, 01GS0850). This study was in part supported by a grant from the German Federal Ministry of Education and Research (BMBF) to the German Center for Diabetes Research (DZD e.V.).

Duality of interest The authors declare that there is no duality of interest associated with this manuscript.

Open Access This article is distributed under the terms of the Creative Commons Attribution Noncommercial License which permits any noncommercial use, distribution, and reproduction in any medium, provided the original author(s) and source are credited.

\section{References}

1. Kopelman PG (2000) Obesity as a medical problem. Nature 404:635-643

2. Marchesini G, Bugianesi E, Forlani G et al (2003) Nonalcoholic fatty liver, steatohepatitis, and the metabolic syndrome. Hepatology 37:917-923

3. Berk PD (2008) Regulatable fatty acid transport mechanisms are central to the pathophysiology of obesity, fatty liver, and metabolic syndrome. Hepatology 48:1362-1376

4. den Boer M, Voshol PJ, Kuipers F, Havekes LM, Romijn JA (2004) Hepatic steatosis: a mediator of the metabolic syndrome. Lessons from animal models. Arterioscler Thromb Vasc Biol 24:644-649

5. Fabbrini E, Sullivan S, Klein S (2010) Obesity and nonalcoholic fatty liver disease: biochemical, metabolic, and clinical implications. Hepatology 51:679-689

6. Korenblat KM, Fabbrini E, Mohammed BS, Klein S (2008) Liver, muscle, and adipose tissue insulin action is directly related to intrahepatic triglyceride content in obese subjects. Gastroenterology 134:1369-1375

7. Postic C, Girard J (2008) Contribution of de novo fatty acid synthesis to hepatic steatosis and insulin resistance: lessons from genetically engineered mice. J Clin Invest 118:829-838

8. Lefkowitch JH, Haythe JH, Regent N (2002) Kupffer cell aggregation and perivenular distribution in steatohepatitis. Mod Pathol 15:699-704

9. Cai D, Yuan M, Frantz DF et al (2005) Local and systemic insulin resistance resulting from hepatic activation of IKK-beta and NFkappaB. Nat Med 11:183-190

10. Kolak M, Westerbacka J, Velagapudi VR et al (2007) Adipose tissue inflammation and increased ceramide content characterize subjects with high liver fat content independent of obesity. Diabetes 56:1960-1968
11. Tsochatzis EA, Papatheodoridis GV, Archimandritis AJ (2009) Adipokines in nonalcoholic steatohepatitis: from pathogenesis to implications in diagnosis and therapy. Mediators Inflamm 2009:831670

12. Lanthier N, Molendi-Coste O, Horsmans Y, van Rooijen N, Cani PD, Leclercq IA (2009) Kupffer cell activation is a causal factor for hepatic insulin resistance. Am J Physiol Gastrointest Liver Physiol 298:G107-G116

13. Obstfeld AE, Sugaru E, Thearle M et al (2010) CCR2 regulates the hepatic recruitment of myeloid cells that promote obesityinduced hepatic steatosis. Diabetes 59:916-925

14. Mazzali M, Kipari T, Ophascharoensuk V, Wesson JA, Johnson R, Hughes J (2002) Osteopontin-a molecule for all seasons. QJM 95:3-13

15. Standal T, Borset M, Sundan A (2004) Role of osteopontin in adhesion, migration, cell survival and bone remodeling. Exp Oncol 26:179-184

16. Xu G, Sun W, He D et al (2005) Overexpression of osteopontin in rheumatoid synovial mononuclear cells is associated with joint inflammation, not with genetic polymorphism. J Rheumatol $32: 410-416$

17. Gomez-Ambrosi J, Catalan V, Ramirez B et al (2007) Plasma osteopontin levels and expression in adipose tissue are increased in obesity. J Clin Endocrinol Metab 92:3719-3727

18. Kiefer FW, Zeyda M, Todoric J et al (2008) Osteopontin expression in human and murine obesity: extensive local upregulation in adipose tissue but minimal systemic alterations. Endocrinology 149:1350-1357

19. Kiefer FW, Zeyda M, Gollinger K et al (2010) Neutralization of osteopontin inhibits obesity-induced inflammation and insulin resistance. Diabetes 59:935-946

20. Nomiyama T, Perez-Tilve D, Ogawa D et al (2007) Osteopontin mediates obesity-induced adipose tissue macrophage infiltration and insulin resistance in mice. J Clin Invest 117:2877-2888

21. Bertola A, Deveaux V, Bonnafous S et al (2009) Elevated expression of osteopontin may be related to adipose tissue macrophage accumulation and liver steatosis in morbid obesity. Diabetes 58:125-133

22. Sahai A, Malladi P, Melin-Aldana H, Green RM, Whitington PF (2004) Upregulation of osteopontin expression is involved in the development of nonalcoholic steatohepatitis in a dietary murine model. Am J Physiol Gastrointest Liver Physiol 287:G264-G273

23. Sahai A, Malladi P, Pan X et al (2004) Obese and diabetic $\mathrm{db} / \mathrm{db}$ mice develop marked liver fibrosis in a model of nonalcoholic steatohepatitis: role of short-form leptin receptors and osteopontin. Am J Physiol Gastrointest Liver Physiol 287:G1035-G1043

24. Kwon HJ, Won YS, Yoon WK, Nam KH, Kim DY, Kim HC (2010) The role of osteopontin in d-galactosamine-induced liver injury in genetically obese mice. Toxicol Appl Pharmacol 242:344-351

25. Neschen S, Morino K, Dong J et al (2007) n-3 Fatty acids preserve insulin sensitivity in vivo in a peroxisome proliferatoractivated receptor-alpha-dependent manner. Diabetes 56:10341041

26. Neschen S, Morino K, Hammond LE et al (2005) Prevention of hepatic steatosis and hepatic insulin resistance in mitochondrial acyl-CoA:glycerol-sn-3-phosphate acyltransferase 1 knockout mice. Cell Metab 2:55-65

27. Haemmerle G, Zimmermann R, Strauss JG et al (2002) Hormonesensitive lipase deficiency in mice changes the plasma lipid profile by affecting the tissue-specific expression pattern of lipoprotein lipase in adipose tissue and muscle. J Biol Chem 277:12946-12952

28. Zeyda M, Saemann MD, Stuhlmeier KM et al (2005) Polyunsaturated fatty acids block dendritic cell activation and function independently of NF-kappaB activation. J Biol Chem 280:1429314301 
29. Kim JB, Wright HM, Wright M, Spiegelman BM (1998) ADD1/ SREBP1 activates PPARgamma through the production of endogenous ligand. Proc Natl Acad Sci U S A 95:4333-4337

30. Yen CL, Stone SJ, Koliwad S, Harris C, Farese RV Jr (2008) Thematic review series: glycerolipids. DGAT enzymes and triacylglycerol biosynthesis. J Lipid Res 49:2283-2301

31. Morino K, Petersen KF, Shulman GI (2006) Molecular mechanisms of insulin resistance in humans and their potential links with mitochondrial dysfunction. Diabetes 55(Suppl 2):S9-S15

32. Dong XC, Copps KD, Guo S et al (2008) Inactivation of hepatic Foxo1 by insulin signaling is required for adaptive nutrient homeostasis and endocrine growth regulation. Cell Metab 8:65-76

33. Puigserver P, Spiegelman BM (2003) Peroxisome proliferatoractivated receptor-gamma coactivator 1 alpha (PGC-1 alpha): transcriptional coactivator and metabolic regulator. Endocr Rev 24:78-90

34. Baffy G (2009) Kupffer cells in non-alcoholic fatty liver disease: the emerging view. J Hepatol 51:212-223

35. Murano I, Barbatelli G, Parisani V et al (2008) Dead adipocytes, detected as crown-like structures, are prevalent in visceral fat depots of genetically obese mice. J Lipid Res 49:1562-1568

36. de Taeye BM, Novitskaya T, McGuinness OP et al (2007) Macrophage TNF-alpha contributes to insulin resistance and hepatic steatosis in diet-induced obesity. Am J Physiol Endocrinol Metab 293:E713-E725

37. Qureshi K, Abrams GA (2007) Metabolic liver disease of obesity and role of adipose tissue in the pathogenesis of nonalcoholic fatty liver disease. World J Gastroenterol 13:3540-3553
38. Seki E, de Minicis S, Osterreicher CH et al (2007) TLR4 enhances TGF-beta signaling and hepatic fibrosis. Nat Med 13:1324-1332

39. Ramaiah SK, Rittling S (2008) Pathophysiological role of osteopontin in hepatic inflammation, toxicity, and cancer. Toxicol Sci 103:4-13

40. Zeyda M, Stulnig TM (2007) Adipose tissue macrophages. Immunol Lett 112:61-67

41. Stienstra R, Saudale F, Duval C et al (2010) Kupffer cells promote hepatic steatosis via interleukin-lbeta-dependent suppression of peroxisome proliferator-activated receptor alpha activity. Hepatology 51:511-522

42. Huang W, Metlakunta A, Dedousis N et al (2010) Depletion of liver Kupffer cells prevents the development of diet-induced hepatic steatosis and insulin resistance. Diabetes 59:347357

43. Kanda H, Tateya S, Tamori Y et al (2006) MCP-1 contributes to macrophage infiltration into adipose tissue, insulin resistance, and hepatic steatosis in obesity. J Clin Invest 116:1494-1505

44. Baggiolini M (1998) Chemokines and leukocyte traffic. Nature 392:565-568

45. Diao H, Kon S, Iwabuchi K et al (2004) Osteopontin as a mediator of NKT cell function in T cell-mediated liver diseases. Immunity 21:539-550

46. Syn WK, Choi SS, Liaskou E, Karaca GF, Agboola KM, Oo YH (2010) Osteopontin is induced by hedgehog pathway activation and promotes fibrosis progression in nonalcoholic steatohepatitis. Hepatology 53:106-115 